\title{
A REFINEMENT OF AN INEQUALITY OF JOHNSON, LOEWY AND LONDON ON NONNEGATIVE MATRICES AND SOME APPLICATIONS.*
}

\author{
THOMAS J. LAFFEY ${ }^{\dagger}$ AND ELEANOR MEEHAN ${ }^{\dagger}$
}

Dedicated to Hans Schneider on the occasion of his seventieth birthday.

Abstract. Let $A$ be an entrywise nonnegative $n \times n$ matrix and let $s_{k}:=\operatorname{trace}\left(A^{k}\right)(k=$ $1,2, \ldots)$. It is shown that if $n$ is odd and $s_{1}=0$, then $(n-1) s_{4} \geq s_{2}^{2}$. The result is applied to show that $\left(3, \frac{1}{2}(1 \pm \sqrt{17}),-2,-2\right)$ is not the spectrum of a nonnegative $5 \times 5$ matrix while $\left(3, \frac{1}{2}(1 \pm \sqrt{17}),-2,-2,0\right)$ is the spectrum of a nonnegative symmetric $6 \times 6$ matrix.

Key words. nonnegative matrix, spectrum, eigenvalues, Johnson-Loewy-London inequalities, symmetric, triangular graph, copositive matrix.

AMS subject classifications. 15A48, 15A18, 05C50, 15A45

1. Introduction. Let $A$ be an entrywise nonnegative $n \times n$ matrix and let

$$
s_{k}:=\operatorname{trace}\left(A^{k}\right), \quad(k=1,2, \ldots) .
$$

The $J L L$-inequalities discovered independently by Loewy and London [10] and Johnson [5] state that

$$
n^{m-1} s_{k m} \geq s_{k}^{m}
$$

for all positive integers $k, m$.

Equality can occur in $(J L L)$ for various $k, m$; for example, if $A^{k}$ is a scalar matrix, then equality holds for all $m$.

In this paper we obtain a refinement of one of the inequalities in a special case. We prove MaIN THEOREM. Let $A$ be an entrywise nonnegative $n \times n$ matrix with $\operatorname{trace}(A)=0$. Then, if $n$ is odd,

$$
(n-1) s_{4} \geq s_{2}^{2},
$$

that is,

$$
(n-1) \operatorname{trace}\left(A^{4}\right) \geq\left(\operatorname{trace}\left(A^{2}\right)\right)^{2} .
$$

This inequality is best possible-for example if $A$ is the matrix

$$
\operatorname{diag}\left(\begin{array}{ll}
0 & 1 \\
1 & 0
\end{array}\right) \oplus \cdots \oplus \operatorname{diag}\left(\begin{array}{ll}
0 & 1 \\
1 & 0
\end{array}\right) \oplus(0)
$$

*Received by the editors on 27 November 1997. Accepted for publication on 24 July 1998. Handling Editor: Daniel Hershkowitz.

${ }^{\dagger}$ Department of Mathematics, University College Dublin, Belfield, Dublin 4, Ireland (laffey@ollamh.ucd.ie, Eleanor.Walsh@itsrv.rtc-limerick.ie) 
then equality occurs. It also fails in general for $n$ even, for example

$$
A=\operatorname{diag}\left(\begin{array}{ll}
0 & 1 \\
1 & 0
\end{array}\right) \oplus \cdots \oplus \operatorname{diag}\left(\begin{array}{ll}
0 & 1 \\
1 & 0
\end{array}\right)
$$

provides a counterexample. In the case $n=5$, this inequality was proved in a number of special cases by Reams [11], [12].

Though the refinement in the $J L L$ inequality is small, it has some important applications. It enables one to show that there exists a list $\left(\lambda_{1}, \ldots, \lambda_{5}\right)$ of real numbers which is not the spectrum of a nonnegative $5 \times 5$ matrix but the list with 0 adjoined, $\left(\lambda_{1}, \ldots, \lambda_{5}, 0\right)$, is the spectrum of a nonnegative symmetric $6 \times 6$ matrix. In [6], Johnson, Loewy and the first author presented a list $\left(\lambda_{1}, \ldots, \lambda_{r}\right)$ of real numbers which forms the spectrum of a nonnegative $r \times r$ matrix but not the spectrum of a nonnegative symmetric $r \times r$ matrix; see also [8]. This was placed in the context of Boyle-Handelman theory [2], [3] and showed that in realizing a given list satisfying the obvious necessary conditions as the nonzero part of the spectrum of a nonnegative matrix $A$, one cannot in general place restrictions on the rank of the zero eigenvalue part of the Jordan form of $A$. However, if $A$ is real symmetric, then its rank is the same as the number of nonzero elements in its spectrum, so the example presented here is significant.

The paper is organized as follows. In Section 1 we consider a quadratic form associated with the triangular graphs. In Section 2 we show how the desired inequality can be formulated in terms of the copositivity of the form. This copositivity is established in Section 3. In Section 4, the result is applied to determine the realizability of certain spectra.

2. The triangular graph. Let $n \geq 5$ be an integer. Let $S$ be the set of all 2 -subsets of $\{1,2, \ldots, n\}$. Define a graph,$n$ as follows:

, ${ }_{n}$ has vertex set $S$ and for $\alpha, \beta \in S, \alpha \beta=\beta \alpha$ is an edge of ${ }_{n}$ if and only if $|\alpha \cap \beta|=1$. The graph, ${ }_{n}$ is known as the triangular graph on $\{1,2, \ldots, n\}$. It is an example of a strongly regular graph (see van Lint and Wilson [9, p. 231]) and its parameters are

$$
\left(\left(\begin{array}{l}
n \\
2
\end{array}\right), 2(n-2), n-2,4\right)
$$

This means that it has $\left(\begin{array}{l}n \\ 2\end{array}\right)$ vertices, each vertex has degree $2(n-2)$ and if $\alpha, \beta$ are distinct vertices, then if $\alpha \beta$ is an edge, there are $n-2$ vertices $\gamma$ incident with both $\alpha$ and $\beta$ while if $\alpha \beta$ is not an edge, there are 4 vertices incident with $\alpha$ and $\beta$. Note also that ${ }_{n}$ is the line graph $L\left(K_{n}\right)$ of the complete graph $K_{n}$ on $n$ vertices; see Cvetkovič, Doob, Sachs [4, p. 169].

Label the vertices of,$n$ by the symbols $1,2, \ldots, N:=\left(\begin{array}{c}n \\ 2\end{array}\right)$, and let $P_{n}$ be its adjacency matrix. By van Lint and Wilson [9, p. 231]

$$
P_{n}^{2}+(4-(n-2)) P_{n}+(4-2(n-2)) I_{N}=4 J_{N},
$$

where $I_{N}$ is the $N \times N$ identity matrix and $J_{N}$ the $N \times N$ matrix with all its entries equal to 1 . The vector with all components equal to 1 is an eigenvector for both $P_{n}$ 
and $J_{N}$ and the corresponding eigenvalue of $P_{n}$ is $2(n-2)$. The other eigenvalues of $J_{N}$ are all 0 and it follows that the remaining eigenvalues of $P_{n}$ satisfy the equation

$$
x^{2}+(4-(n-2)) x+4-2(n-2)=0 .
$$

So they are $n-4$ and -2 and the corresponding multiplicities are $(n-1)$ and $\left(\begin{array}{c}n-1 \\ 2\end{array}\right)-1$ (as determined from the equation trace $\left(P_{n}\right)=0$ ). [The fact that the eigenvalues of $P_{n}$ are integers is related to the fact that the $\mathbf{Z}$-span of $I, J_{N}, P_{n}$ is a ring (the Bose-Messner algebra of , $n$ [9, pp. 234-235])].

Note also that since, $n$ is the line graph $L\left(K_{n}\right)$,

$$
P_{n}=R_{n}^{T} R_{n}-2 I_{N}
$$

where $R_{n}$ is the incidence matrix of the graph $K_{n}$ (where the edges of $K_{n}$ are labeled consistently with our labeling of the vertices of $P_{n}$ ).

Consider the quadratic form

$$
\begin{aligned}
Q:=\left(x_{1} \cdots x_{N}\right) P_{n}\left(\begin{array}{c}
x_{1} \\
x_{2} \\
\vdots \\
x_{N}
\end{array}\right) & =\left(x_{1} \cdots x_{N}\right) R^{T} R\left(\begin{array}{c}
x_{1} \\
x_{2} \\
\vdots \\
x_{N}
\end{array}\right)-2 \sum_{i=1}^{N} x_{i}^{2} \\
& =\Omega_{1}^{2}+\cdots+\Omega_{n}^{2}-2 \sum_{i=1}^{N} x_{i}^{2}
\end{aligned}
$$

where $\Omega_{i}=x_{i_{j_{i}(1)}}+\cdots+x_{i_{j_{i}(n-1)}}$ and $j_{i}(1), \ldots, j_{i}(n-1)$ are the edges of $K_{n}$ containing the vertex $i(i=1,2, \ldots, n)$. Thus for $i \neq k, \Omega_{i}$ and $\Omega_{k}$ involve exactly one common symbol. We show in the next section that the problem of minimizing the form

$$
\left(x_{1} \cdots x_{N}\right)\left(P_{n}+I\right)\left(\begin{array}{c}
x_{1} \\
x_{2} \\
\vdots \\
x_{N}
\end{array}\right)=\Omega_{1}^{2}+\cdots+\Omega_{n}^{2}-\sum_{i=1}^{N} x_{i}^{2}
$$

over $\mathcal{D}:=\left\{\left(x_{1}, \ldots, x_{N}\right) \mid x_{i} \geq 0\right.$ for all $i$ and $\left.\sum_{i=1}^{N} x_{i}=1\right\}$ arises in the proof of the main theorem.

3. Nonnegative matrices of trace 0 . Let $A=\left(a_{i j}\right)$ be a nonnegative $n \times n$ matrix of trace 0 and let $s_{k}:=\operatorname{trace}\left(A^{k}\right)$ for $k=1,2, \ldots, n$. Note that

$$
s_{2}=\Omega_{1}+\cdots+\Omega_{n}
$$

where

$$
\Omega_{i}=\sum_{\substack{j=1 \\ j \neq i}}^{n} a_{i j} a_{j i} \quad(i=1,2, \ldots, n)
$$


Write $y_{1}=a_{12} a_{21}, y_{2}=a_{13} a_{31}, \ldots, y_{n-1}=a_{1 n} a_{n 1}, y_{n}=a_{23} a_{32}, y_{n+1}=a_{24} a_{42}, \ldots$, $y_{N}=a_{n-1 n} a_{n n-1}$ where $N:=\left(\begin{array}{c}n \\ 2\end{array}\right)$.

We now consider $s_{4}=$ trace $\left(A^{4}\right)$. The diagonal terms of $A^{2}$ contribute $\Omega_{1}^{2}+\cdots \Omega_{n}^{2}$ to $s_{4}$. We observe that this contribution is a quadratic form in $y_{1}, y_{2}, \ldots, y_{N}$. Thus we are led to consider that part of $s_{4}$ which can be expressed as a quadratic form in $y_{1}, \ldots, y_{N}$. Since trace $(A)=0$, all diagonal entries of $A$ are 0 and thus $s_{4}$ is a sum of terms of the form $a_{r_{1} r_{2}} a_{r_{2} r_{3}} a_{r_{3} r_{4}} a_{r_{4} r_{1}}$, where either all the $r_{i}$ are distinct or it equals a term of the form $a_{p q} a_{q p} a_{r s} a_{s r}$.

The 4-cycle type $a_{r_{1} r_{2}} a_{r_{2} r_{3}} a_{r_{3} r_{4}} a_{r_{4} r_{1}}$ with $r_{1}, r_{2}, r_{3}, r_{4}$ distinct does not give a quadratic form in $y_{1}, \ldots, y_{N}$.

Consider a term of the form

$$
a_{p q} a_{q p} a_{r s} a_{s r}
$$

where $p \neq q, r \neq s$. If $\{r, s\}=\{p, q\}$, this term only arises in the component $\Omega_{1}^{2}+\cdots+\Omega_{n}^{2}$. Suppose $\{r, s\} \neq\{p, q\}$. If $\{r, s\} \cap\{p, q\}$ is empty, this term cannot occur in $s_{4}$ at all. Hence we are led to consider the occurrence of terms $a_{p q} a_{q p} a_{r s} a_{s r}$ in which

$$
|\{p, q\} \cap\{r, s\}|=1
$$

(where $|\cdot|$ denotes cardinality).

Suppose $p=r$. Then such a term occurs in $s_{4}-\left(\Omega_{1}^{2}+\cdots+\Omega_{n}^{2}\right)$ in the following ways: in the contribution of the $(q, s)$ term of $A^{2}$ and the $(s, q)$ term of $A^{2}$ to the $(q, q)$ element of $A^{4}$ and also to the $(s, s)$ element of $A^{4}$ and in no other way, so its coefficient in $s_{4}-\left(\Omega_{1}^{2}+\cdots+\Omega_{n}^{2}\right)$ is 2 .

If $p=s$, then the term arises in the $(q, q)$ and $(r, r)$ terms contribution to $s_{4}-$ $\left(\Omega_{1}^{2}+\cdots+\Omega_{n}^{2}\right)$ and not in any other term.

Similarly the term occurs with coefficient 2 in $s_{4}-\left(\Omega_{1}^{2}+\cdots+\Omega_{n}^{2}\right)$ if $\{q\}=$ $\{p, q\} \cap\{r, s\}$.

Thus we find that

$$
s_{4} \geq \Omega_{1}^{2}+\cdots+\Omega_{n}^{2}+2 \sum y_{l} y_{m}
$$

where the sum is over all $\{l, m\}$ such that if $y_{l}=a_{p q} a_{q p}$ and $y_{m}=a_{r s} a_{s r}$, then $|\{p, q, r, s\}|=3$.

Thus in the notation of Section 1,

$$
s_{4} \geq \Omega_{1}^{2}+\cdots+\Omega_{n}^{2}+\left(y_{1} \cdots y_{N}\right) P_{n}\left(\begin{array}{c}
y_{1} \\
\vdots \\
y_{N}
\end{array}\right) \text {. }
$$

But note that

$$
\Omega_{1}^{2}+\cdots+\Omega_{n}^{2}=\left(y_{1} \cdots y_{N}\right) R^{T} R\left(\begin{array}{c}
y_{1} \\
\vdots \\
y_{N}
\end{array}\right)
$$


Since $P_{n}=R^{T} R-2 I_{N}$, we have

$$
s_{4} \geq 2\left(\Omega_{1}^{2}+\cdots+\Omega_{n}^{2}\right)-2 \sum_{i=1}^{N} y_{i}^{2} .
$$

To prove the theorem, it suffices to show that

$$
2(n-1)\left(\Omega_{1}^{2}+\cdots+\Omega_{n}^{2}\right)-2(n-1) \sum_{i=1}^{N} y_{i}^{2} \geq s_{2}^{2}=4\left(y_{1}+\cdots+y_{N}\right)^{2} .
$$

4. An optimization problem. Motivated by Section 2, we formulate the following question. Let $\mathcal{D}=\left\{\left(y_{1}, \ldots, y_{N}\right) \mid y_{i} \geq 0\right.$ for all $i$ and $\left.\sum_{i=1}^{N} y_{i}=1\right\}$ where $n$ is an odd integer $\geq 3$ and $N=\left(\begin{array}{c}n \\ 2\end{array}\right)$. Let $\Lambda_{1}, \Lambda_{2}, \ldots, \Lambda_{n}$ be a collection of subsets of $\left\{y_{1}, y_{2}, \ldots, y_{N}\right\}$ satisfying the following properties.

1. Each $\Lambda_{i}$ contains $n-1 y_{i} \mathrm{~s}$

2. $\left|\Lambda_{i} \cap \Lambda_{j}\right|=1$ for all $1 \leq i \neq j \leq n$

3 . Each $y_{i}$ belongs to exactly two of the $\Lambda_{j} \mathrm{~s}$.

Let $\Omega_{i}$ be the sum of the elements of $\Lambda_{i}$ and let

$$
\Phi\left(y_{1}, \ldots, y_{N}\right):=\Omega_{1}^{2}+\cdots+\Omega_{n}^{2}-\sum_{i=1}^{N} y_{i}^{2} .
$$

The problem is to determine the global minimum of $\Phi\left(y_{1}, \ldots, y_{N}\right)$ as $y$ runs through $\mathcal{D}$. Since $\Phi$ is a continuous function on the compact set $\mathcal{D}$ the global minimum exists. We now suppose $y_{1}, \ldots, y_{N}$ have been chosen so that

(1) $\Phi\left(y_{1}, \ldots, y_{N}\right)$ is the global minimum of $\Phi$ on $\mathcal{D}$

(2) The number of nonzero $y_{i}$ is least possible subject to condition (1).

We first establish the following result.

CLAIM 1. Assume the notation is chosen so that $y_{1}=\max _{1 \leq i \leq N} y_{i}$ and that

$y_{1} \in \Lambda_{1} \cap \Lambda_{2}$. Then $\Omega_{1}=\Omega_{2}=y_{1}$ (that is, all the $y_{i}$ s occurring in $\left(\Lambda_{1} \cup \Lambda_{2}\right) \backslash\left\{y_{1}\right\}$ are zero).

Proof. For suppose $\Omega_{1}$ contains an element $y_{j}(j \neq 1)$ with $y_{j} \neq 0$ and choose $y_{j}$ least possible subject to this.

Define $y_{1}^{\prime}=y_{1}+y_{j}, y_{j}^{\prime}=0$.

Let $\Lambda_{1}=\left\{y_{1}, y_{j}, y_{h_{3}}, \ldots, y_{h_{n-1}}\right\}, \Lambda_{2}=\left\{y_{1}, y_{r_{2}}, \ldots, y_{r_{n-1}}\right\}$ and note $y_{j} \notin \Lambda_{2}$ by property 2. Put $\Lambda_{1}^{\prime}=\left\{y_{1}^{\prime}, y_{j}^{\prime}, y_{h_{3}}, \ldots, y_{h_{n-1}}\right\}, \Lambda_{2}^{\prime}=\left\{y_{1}^{\prime}, y_{r_{2}}, \ldots, y_{r_{n-1}}\right\}$. Now $y_{j}$ is contained in some $\Lambda_{i}(i>1)$, say $y_{j} \in \Lambda_{3}$. Define

$$
\Lambda_{3}^{\prime}=\left(\Lambda_{3} \backslash\left\{y_{j}\right\}\right) \cup\left\{y_{j}^{\prime}\right\} .
$$

Define $\Lambda_{k}^{\prime}=\Lambda_{k}$ for all $k>3$. Consider the effect of this change on

$$
\Omega_{1}^{2}+\cdots \Omega_{n}^{2}-\sum_{i=1}^{N} y_{i}^{2}
$$


Note that $\Omega_{1}^{\prime}=\Omega_{1}$ and that $\left(\Omega_{2}^{\prime}\right)^{2}$ is increased (over $\Omega_{2}^{2}$ ) by

$$
y_{j}^{2}+2 y_{j}\left(y_{1}+y_{r_{2}}+\cdots+y_{r_{n}}\right)=y_{j}^{2}+2 y_{j} \Omega_{2} .
$$

Also $\left(\Omega_{3}^{\prime}\right)^{2}$ is reduced (over $\Omega_{3}^{2}$ ) by

$$
y_{j}^{2}+2 y_{j}\left(\Omega_{3}-y_{j}\right)
$$

Also $y_{1}^{2}+y_{j}^{\prime 2}+\sum_{t \neq 1, j} y_{t}^{2}$ is increased (over $\sum_{i=1}^{N} y_{i}^{2}$ ) by $2 y_{1} y_{j}$.

Thus the overall effect on the function $\Phi$ is an increase of

$$
2 y_{j}\left(\Omega_{2}-y_{1}\right)-2 y_{j}\left(\Omega_{3}-y_{j}\right)=2 y_{j}\left[\left(\Omega_{2}-y_{1}\right)-\left(\Omega_{3}-y_{j}\right)\right] .
$$

It follows that $\Omega_{2}-y_{1} \geq \Omega_{3}-y_{j}$. But if equality occurs here, then $\Phi$ is unchanged while the number of nonzero $y_{i}$ 's has been decreased by 1 , contrary to hypothesis (2) above. Hence we have

$$
\Omega_{2}-y_{1}>\Omega_{3}-y_{j} .
$$

Consider the following swap of the original $\Lambda_{i}$.

Leave $\Lambda_{1}, \Lambda_{4}, \ldots, \Lambda_{n}$ as before.

Put

$$
\begin{aligned}
& \Lambda_{2}^{\prime \prime}=\left(\Lambda_{3} \backslash\left\{y_{j}\right\}\right) \cup\left\{y_{1}\right\} \\
& \Lambda_{3}^{\prime \prime}=\left(\Lambda_{2} \backslash\left\{y_{1}\right\}\right) \cup\left\{y_{j}\right\}
\end{aligned}
$$

Note that the system

$$
\Lambda_{1}, \Lambda_{2}^{\prime \prime}, \Lambda_{3}^{\prime \prime}, \Lambda_{4}, \ldots, \Lambda_{n}
$$

satisfies the hypothesis and that the sum $\sum y_{i}^{2}$ is unchanged and the overall change in $\Phi$ is to add

$$
2 y_{1}\left(\Omega_{3}-y_{j}\right)+2 y_{j}\left(\Omega_{2}-y_{1}\right)=2\left(y_{1}-y_{j}\right)\left[\left(\Omega_{3}-y_{j}\right)-\left(\Omega_{2}-y_{1}\right)\right] .
$$

Since $\Phi$ achieves its global minimum for these $y_{i}$ 's, it follows that this must be nonnegative.

If $y_{1}-y_{j}>0$, this implies that

$$
\left(\Omega_{3}-y_{j}\right)-\left(\Omega_{2}-y_{1}\right) \geq 0,
$$

contradicting $(*)$ above. Hence $y_{1}=y_{j}$ and $\Omega_{2}>\Omega_{3}$. But now we can reverse the roles of $y_{1}$ and $y_{j}$ and define $y_{1}^{\prime}=0$ and $y_{j}^{\prime}=y_{1}+y_{j}$. The argument then yields $\Omega_{2}<\Omega_{3}$, thus yielding the desired contradiction. $\mathrm{Q}$

CLAIM 2. The global minimum is achieved by an assignment in which each $\Lambda_{i}$ has at most one nonzero $y_{i}$. 
Proof. By Claim 1, we may assume $\Lambda_{1}=\Lambda_{2}=\left\{y_{1}\right\}$, where we assume $y_{1}=$ $\max \left\{y_{1}, \ldots, y_{N}\right\}$. The Claim is now clear if $n=3$. Suppose $n>3$. Using our hypotheses, we may write

$$
\Lambda_{1}=\left\{y_{1}, y_{2}, \ldots, y_{n-1}\right\} \quad \text { and } \quad \Lambda_{2}=\left\{y_{1}, y_{n}, y_{n+1}, \ldots, y_{2 n-3}\right\} \text {, }
$$

and $y_{2}=0, \ldots, y_{n-1}=0, y_{n}=0, \ldots, y_{2 n-3}=0$. Note that $y_{2}, \ldots, y_{n-1}$ belong to distinct members of the list $\Lambda_{3}, \ldots, \Lambda_{n}$ and thus each $\Lambda_{i}(i=3, \ldots, n)$ contains exactly one of $y_{2}, \ldots, y_{n-1}$. Similarly $\Lambda_{3}, \ldots, \Lambda_{n}$ contains exactly one of $y_{n}, \ldots, y_{2 n-3}$. But now deleting those elements from $\Lambda_{3}, \ldots, \Lambda_{n}$ leads to a list $\Lambda_{3}^{\prime}, \ldots, \Lambda_{n}^{\prime}$ of $n-2$ subsets of $\left\{y_{2 n-2}, \ldots, y_{N}\right\}$ which satisfy our hypotheses with $n$ replaced by $n-2$ (and $\sum_{i=1}^{N} y_{i}=1$ replaced by $\left.\sum_{i=2 n-2}^{N} y_{i}=1-2 y_{1}\right)$.

So now we can use induction on $n$ to conclude that the minimum contribution from these subsets occurs for an arrangement in which each of $\Lambda_{3}^{\prime}, \ldots, \Lambda_{n}^{\prime}$ contains at most one nonzero element. This establishes Claim 2.

Since $n$ is odd, Claim 2 implies that one of the $\Lambda_{k} \mathrm{~s}$ consists of zeros since the nonzero elements pair off as $\left(\left\{y_{1}\right\},\left\{y_{1}\right\}\right),\left(\left\{y_{2}\right\},\left\{y_{2}\right\}\right), \ldots$ Thus the problem of minimizing $\Phi$ in this case reduces to: Minimize $2\left(y_{1}^{2}+y_{2}^{2}+\cdots+y_{k}^{2}\right)-\left(y_{1}^{2}+\cdots+y_{k}^{2}\right)$ (where $k=\frac{n-1}{2}$ ) subject to $y_{i} \geq 0$ and $\sum_{i=1}^{k} y_{i}=1$.

But for any real numbers $a, b$

$$
(a-b)^{2}+(a+b)^{2}>2 a^{2}
$$

if $b \neq 0$.

Hence at the global minimum, all $y_{i}$ must be equal, and thus they must equal $\frac{1}{k}$ and the global minimum of $\Phi$ is

$$
\frac{k}{k^{2}}=\frac{1}{k}=\frac{2}{n-1} \text {. }
$$

Proof of $(E)$. The proof of $(E)$ reduces to showing that

$$
2(n-1)\left[\left(\Omega_{1}^{2}+\cdots+\Omega_{n}^{2}\right)-\sum_{i=1}^{N} y_{i}^{2}\right] \geq 4\left(y_{1}+\cdots+y_{N}\right)^{2}
$$

for $n$ odd, and all $y_{1} \geq 0, \ldots, y_{N} \geq 0$. We may assume $y_{1}+\cdots+y_{N}=1$, otherwise replace each $y_{i}$ by $y_{i} /\left(\sum_{j=1}^{N} y_{j}\right)$.

But now by the optimization result,

$$
2(n-1)\left[\Omega_{1}^{2}+\cdots+\Omega_{n}^{2}-\sum_{i=1}^{N} y_{i}^{2}\right] \geq \frac{2(n-1)}{k}=4
$$


since $\frac{1}{k}$ is the global minimum of

$$
\Omega_{1}^{2}+\cdots+\Omega_{n}^{2}-\sum_{i=1}^{N} y_{i}^{2}
$$

This proves $(E)$ and completes the proof of the Main Theorem for $n \geq 3$. Note that we have equality in the theorem when $n=3$.

5. Applications. 1. Consider the cubic multigraph (Cvetkovic et al [4, (4.15) p. 309]) with adjacency matrix

$$
A:=\left(\begin{array}{llllll}
0 & 1 & 1 & 1 & 0 & 0 \\
1 & 0 & 2 & 0 & 0 & 0 \\
1 & 2 & 0 & 0 & 0 & 0 \\
1 & 0 & 0 & 0 & 1 & 1 \\
0 & 0 & 0 & 1 & 0 & 2 \\
0 & 0 & 0 & 1 & 2 & 0
\end{array}\right)
$$

The spectrum of $A$ is

$$
\left(3, \frac{1}{2}(1+\sqrt{17}), \frac{1}{2}(1-\sqrt{17}),-2,-2,0\right)
$$

and $4 \operatorname{trace}\left(A^{4}\right)-\left(\operatorname{trace}\left(A^{2}\right)\right)^{2}=-28$ so $\left(3, \frac{1}{2}(1+\sqrt{17}), \frac{1}{2}(1-\sqrt{17}),-2,-2\right)$ is not the spectrum of a nonnegative $5 \times 5$ matrix

2. The list $(10,8,-7,-6,-5)$ is not the spectrum of a nonnegative $5 \times 5$ matrix since

$$
4 s_{4}-s_{2}^{2}=-1404 .
$$

This list was proposed as a test spectrum by R. Loewy since it satisfied all previously known necessary conditions for realizability by a nonnegative matrix.

3 . The list $(3,3,-2,-2,-2)$ has been used as an example by several authors. It satisfies the $J L L$ inequalities but is not realizable as the spectrum of a nonnegative matrix $A$. For if such an $A$ exists, since the Perron eigenvalue 3 is repeated, $A$ would have to be reducible under permutation similarity and thus the list would have to be partitionable into two lists which are separately realizable and this is clearly impossible. Note that its unrealizability also follows from the Main Theorem since $4 s_{4}-s_{2}^{2}=-60$. One can seek the least positive number $t$ for which

$$
(3+t, 3-t,-2,-2,-2)
$$

is realizable. A necessary condition is that $4 s_{4}-s_{2}^{2} \geq 0$, that is

$$
\begin{gathered}
t^{4}+78 t^{2}-15 \geq 0 \\
t \geq t_{0}:=\sqrt{16 \sqrt{6}-39}=0.43799 \ldots
\end{gathered}
$$


But for such a real number $t$, the matrix

$$
\left(\begin{array}{ccccc}
0 & 1 & 0 & 0 & 0 \\
\frac{15+t^{2}}{2} & 0 & 1 & 0 & 0 \\
0 & 0 & 0 & 1 & 0 \\
0 & 0 & 0 & 0 & 1 \\
v & \frac{\left(t^{4}+78 t^{2}-15\right)}{4} & 10+6 t^{2} & \frac{15+t^{2}}{2} & 0
\end{array}\right)
$$

where $v=-72+8 t^{2}+\left(15+t^{2}\right)\left(5+3 t^{2}\right)=3 t^{4}+58 t^{2}+3$ has the spectrum $(3+t, 3-$ $t,-2,-2,-2)$. By Guo Wuwen $[13],(3+\beta, 3,-2,-2,-2)$ is therefore the spectrum of a nonnegative matrix for all $\beta \geq 2 t_{0}=0.87598 \cdots$

This is perhaps surprising in view of a generally held view that $\beta \geq 1$ was best possible here. The difficult question of finding the best possible bound on $\beta$ will be considered elsewhere.

4. A real symmetric $n \times n$ matrix $A$ is called copositive if $x^{T} A x \geq 0$ for all vectors $x$ with nonnegative entries; see [1], [7]. The class of copositive matrices obviously includes the class of positive semi-definite and positive definite matrices and also the class of (entrywise) nonnegative symmetric matrices. Furthermore it is clear that if $A=P+N$ when $P$ is a positive semi-definite real symmetric matrix and $N$ a nonnegative real symmetric matrix, then $A$ is copositive, and a question arose as to whether conversely every copositive matrix is expressible as such a sum. A counterexample to this was constructed by A. Horn [7, (16.2)] and it is interesting to note that with a suitable ordering of the vertices, his example coincides with the leading principal $5 \times 5$ submatrix of $2\left(I+P_{5}\right)-J_{10}$. But, by $(\mathrm{E})$ above, for nonnegative $x_{1}, \ldots, x_{10}$

$$
\begin{aligned}
\left(x_{1}, \ldots, x_{10}\right)^{T} 2\left(I+P_{5}\right)\left(\begin{array}{c}
x_{1} \\
\vdots \\
x_{10}
\end{array}\right) & \geq\left(x_{1}+\cdots+x_{10}\right)^{2} \\
& =\left(x_{1}, \ldots, x_{10}\right)^{T} J_{10}\left(\begin{array}{c}
x_{1} \\
\vdots \\
x_{10}
\end{array}\right)
\end{aligned}
$$

Hence $2\left(I+P_{5}\right)-J_{10}$ is copositive.

It then follows from Horn's argument that $2\left(I+P_{5}\right)-J_{10}$ is not of the form $X+Y$ where $X$ is a nonnegative symmetric matrix and $Y$ is a positive semi-definite real symmetric matrix. The cone of copositive $n \times n$ matrices is the dual of the cone of completely positive matrices; see [1], [7].

Acknowledgement. The authors wish to thank the two referees for very carefully reading the paper and for their helpful suggestions which have improved its readability.

\section{REFERENCES}

[1] A. Berman, Complete positivity. Linear Algebra and its Applications, 107:57-63, 1988. 
[2] M. Boyle and D. Handelman, The spectra of nonnegative matrices via symbolic dynamics, Annals of Mathematics, 133:249-316, 1991.

[3] M. Boyle and D. Handelman, Algebraic shift equivalence and primitive matrices, Transactions of the American Mathematics Society, 336:121-50, 1993.

[4] D. M. Cvetkovič, M. Doob and H. Sachs, Spectra of graphs-theory and applications. Academic Press, New York, 1982.

[5] C. R. Johnson, Row stochastic matrices that are similar to doubly stochastic matrices. Linear and Multilinear Algebra, 10:113-120, 1981.

[6] C R Johnson, T. J. Laffey and R. Loewy, The real and the symmetric nonnegative inverse eigenvalue problems are different, Proceedings of the American Mathematics Society, 124:36473651,1996

[7] M. Hall, Combinatorial Theory, Wiley, New York, 1986.

[8] T. J. Laffey, Inverse eigenvalue problems for matrices, Proceedings of the Royal Irish Academy 95A (Supplement):81-88, 1995.

[9] J. H. van Lint and R. M. Wilson, A course in combinatorics. Cambridge University Press, Cambridge, 1992.

[10] R. Loewy and D. London, A note on the inverse eigenvalue problems for nonnegative matrices. Linear and Multilinear Algebra, 6:83-90, 1978.

[11] R. Reams, Topics in Matrix Theory. Thesis presented for the degree of Ph.D., National University of Ireland, Dublin, 1994.

[12] R. Reams, An inequality for nonnegative matrices and the inverse eigenvalue problem. Linear and Multilinear Algebra, 41:367-375, 1996.

[13] Guo Wuwen, An inverse eigenvalue problem for nonnegative matrices. Linear Algebra and its Applications, 249:67-78, 1996. 